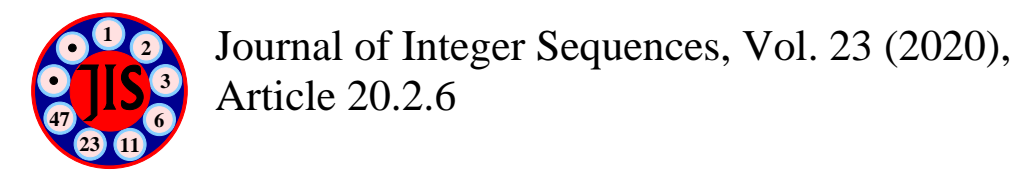

\title{
Two New Explicit Formulas for the Even-Indexed Bernoulli Numbers
}

\author{
Sumit Kumar Jha \\ International Institute of Information Technology \\ Hyderabad-500 032 \\ India \\ kumarjha.sumit@research.iiit.ac.in
}

\begin{abstract}
We give two new explicit formulas for the even-indexed Bernoulli numbers in terms of the Stirling numbers of the second kind.
\end{abstract}

\section{Introduction}

Definition 1. The Bernoulli numbers $B_{n}$ can be defined by the following generating function:

$$
\frac{t}{e^{t}-1}=\sum_{n \geq 0} \frac{B_{n} t^{n}}{n !},
$$

where $|t|<2 \pi$.

Definition 2. The Stirling number of the second kind, denoted by $S(n, m)$, is the number of ways of partitioning a set of $n$ elements into $m$ nonempty sets.

There are many explicit formulas known for the Bernoulli numbers [1, 9, 4, 5, 6]. For example, all of the formulas below express the Bernoulli numbers explicitly in terms of the 
Stirling numbers of the second kind:

$$
\begin{aligned}
B_{r} & =\sum_{k=1}^{r}(-1)^{k} \cdot k ! \cdot \frac{S(r, k)}{k+1}, \\
B_{r} & =\sum_{k=1}^{r}(-1)^{k-1} \cdot(k-1) ! \cdot \frac{S(r, k)}{k+1}, \\
B_{r+1} & =\sum_{k=1}^{r} \frac{(-1)^{k-1} k ! S(r, k)}{(k+1)(k+2)}, \\
B_{r} & =\frac{r}{1-2^{r}} \sum_{k=1}^{r-1} \frac{(-1)^{k} k ! S(r-1, k)}{2^{k+1}}, \\
B_{r+1} & =\frac{(-1)^{r} \cdot(r+1) \cdot 2^{r-1}}{2^{r+1}-1} \sum_{k=1}^{r} \frac{(-1)^{k} k ! S(r, k)}{k+1} \cdot 2^{-2 k}\left(\begin{array}{c}
2 k \\
k
\end{array}\right), \\
B_{r} & =\sum_{i=0}^{r}(-1)^{i} \frac{\left(\begin{array}{c}
r+1 \\
i+1
\end{array}\right)}{\left(\begin{array}{c}
r+i \\
i
\end{array}\right)} S(r+i, i), \\
B_{r+1} & =-\frac{r+1}{4\left(1+2^{-(r+1)}\left(1-2^{-r}\right)\right)}\left(\sum_{k=1}^{r}(-1)^{k} \cdot \frac{S(r, k)}{k+1} \cdot\left(\frac{3}{4}\right)^{(k)}+4^{-r} E_{r}\right),
\end{aligned}
$$

where $\left(E_{r}\right)$ denotes the Euler numbers defined by the following generating function:

$$
\frac{1}{\cosh t}=\frac{2}{e^{t}+e^{-t}}=\sum_{n=0}^{\infty} \frac{E_{n}}{n !} \cdot t^{n} .
$$

In the following section, we derive two new explicit formulas for the even-indexed Bernoulli numbers in terms of the Stirling numbers of the second kind.

\section{Main results}

Our main results are the following.

Theorem 3. For all positive integers $r$ we have

$$
B_{2 r}=\frac{-4 r}{3\left(3-3^{1-2 r}\right)} \sum_{k=1}^{2 r-1}(-1)^{k} \frac{S(2 r-1, k)}{k+1}\left(\frac{2}{3}\right)^{(k)}
$$

and

$$
B_{2 r}=\frac{2 r}{3\left(\left(1-2^{1-2 r}\right)\left(1-3^{1-2 r}\right)-2\right)} \sum_{k=1}^{2 r-1}(-1)^{k} \frac{S(2 r-1, k)}{k+1}\left(\frac{5}{6}\right)^{(k)}
$$


where $S(2 r-1, k)$ denotes the Stirling numbers of the second kind, and

$$
x^{(n)}=x(x+1)(x+2) \cdots(x+n-1)
$$

denotes the rising factorial.

To prove the above result, we first recall the following fact

Theorem 4. [10] The nth Bernoulli polynomial, $B_{n}(t)$, defined by

$$
B_{n}(t)=\sum_{j=0}^{n}\left(\begin{array}{l}
n \\
j
\end{array}\right) B_{n-j} t^{j}
$$

takes the following 'special' values at certain rational numbers with small denominators

$$
\begin{gathered}
B_{n}(1)=B_{n}(0)=B_{n} \quad \text { for } n \geq 1, \\
B_{n}\left(\frac{1}{2}\right)=\left(2^{1-n}-1\right) B_{n}, \\
B_{2 n}\left(\frac{1}{4}\right)=B_{2 n}\left(\frac{3}{4}\right)=\frac{2-2^{2 n}}{4^{2 n}} B_{2 n}, \\
B_{2 n}\left(\frac{1}{3}\right)=B_{2 n}\left(\frac{2}{3}\right)=\frac{3-3^{2 n}}{2 \cdot 3^{2 n}} B_{2 n}, \\
B_{2 n}\left(\frac{1}{6}\right)=B_{2 n}\left(\frac{5}{6}\right)=\frac{\left(2-2^{2 n}\right)\left(3-3^{2 n}\right)}{2 \cdot 6^{2 n}} B_{2 n} .
\end{gathered}
$$

Remark 5. Granville and Sun [2] noted that, "It is not known if $B_{n}(a / q)$ has as simple a 'closed form' for any other rational $a / q$ with $1 \leq a \leq q-1$ and $(a, q)=1$, though this has long been considered an interesting question."

Our proof also requires the following result.

Lemma 6. For all $0<t<1$ and $l \in \mathbb{N}$ we have

$$
\int_{0}^{\infty} x^{t-1} \frac{\mathrm{Li}_{-l}(-x)}{1+x} d x=\frac{\pi}{\sin t \pi}\left(\frac{B_{l+1}(1-t)-B_{l+1}}{l+1}\right),
$$

where $\operatorname{Li}_{-l}(-x)$ is the negative polylogarithm function, and $B_{r}(1-t)$ denotes the Bernoulli polynomial.

Proof. Consider the following generating function [8]

$$
\frac{\mathrm{Li}_{-r}(-x)}{1+x}=\sum_{n=0}^{\infty} S_{r}(n)(-x)^{n}
$$


where $S_{r}(n)=\sum_{k=1}^{n} k^{r}$ for $n \geq 1$, and $S_{r}(0)=0$.

We use Ramanujan's master theorem (RMT) from [3] that states that

$$
\int_{0}^{\infty} x^{t-1}\left\{\phi(0)-x \phi(1)+x^{2} \phi(2)-\cdots\right\} d x=\frac{\pi}{\sin t \pi} \phi(-t)
$$

where the integral is convergent for $0<\Re(t)<1$, and after certain conditions are satisfied by $\phi$.

Using RMT with Eq. (8) gives us Eq. (7).

Proof of Theorem 3. Substituting $t=2 / 3$ and $l=2 r-1$ in Eq. (7) gives us

$$
\int_{0}^{\infty} x^{-1 / 3} \frac{\operatorname{Li}_{1-2 r}(-x)}{1+x} d x=\frac{2 \pi}{\sqrt{3}}\left(\frac{B_{2 r}(1 / 3)-B_{2 r}}{2 r}\right) .
$$

We use the following representation from the note [7]

$$
\mathrm{Li}_{1-2 r}(-x)=\sum_{k=1}^{2 r-1} k ! S(2 r-1, k)\left(\frac{1}{1+x}\right)^{k+1}(-x)^{k}
$$

to conclude that

$$
\begin{aligned}
\int_{0}^{\infty} x^{-1 / 3} \frac{\operatorname{Li}_{1-2 r}(-x)}{1+x} d x= & \sum_{k=1}^{2 r-1}(-1)^{k} \cdot k ! S(2 r-1, k) \int_{0}^{\infty} \frac{x^{k-1 / 3}}{(1+x)^{k+2}} d x \\
= & \sum_{k=1}^{2 r-1}(-1)^{k} \cdot k ! S(2 r-1, k) \frac{\Gamma(k+2 / 3) \Gamma(4 / 3)}{\Gamma(k+2)} \\
= & \sum_{k=1}^{2 r-1}(-1)^{k} \cdot \frac{S(2 r-1, k)}{k+1}\left(\frac{2}{3}\right)^{(k)} \cdot \frac{2 \pi}{3 \sqrt{3}},
\end{aligned}
$$

where $\Gamma(\cdot)$ denotes the Gamma function. Recalling Eq. (5) we have

$$
B_{2 r}(1 / 3)-B_{2 r}=-\left(3-3^{1-2 r}\right) \frac{B_{2 r}}{2} .
$$

Now we can readily conclude Eq. (2).

To prove Eq. (3) we substitute $t=5 / 6$ and $l=2 r-1$ in the Eq. (7) to get

$$
\int_{0}^{\infty} x^{-1 / 6} \frac{\operatorname{Li}_{1-2 r}(-x)}{1+x} d x=2 \pi\left(\frac{B_{2 r}(1 / 6)-B_{2 r}}{2 r}\right) .
$$


The representation (9) also lets us to conclude that

$$
\begin{aligned}
\int_{0}^{\infty} x^{-1 / 6} \frac{\operatorname{Li}_{1-2 r}(-x)}{1+x} d x & =\sum_{k=1}^{2 r-1}(-1)^{k} \cdot k ! S(2 r-1, k) \int_{0}^{\infty} \frac{x^{k-1 / 6}}{(1+x)^{k+2}} d x \\
& =\sum_{k=1}^{2 r-1}(-1)^{k} \cdot k ! S(2 r-1, k) \frac{\Gamma(k+5 / 6) \Gamma(7 / 6)}{\Gamma(k+2)} \\
& =\sum_{k=1}^{2 r-1}(-1)^{k} \cdot \frac{S(2 r-1, k)}{k+1}\left(\frac{5}{6}\right)^{(k)} \cdot \frac{\pi}{3} .
\end{aligned}
$$

Recalling Eq. (6) we have

$$
B_{2 r}(1 / 6)-B_{2 r}=\frac{B_{2 r}}{2}\left(\left(1-2^{1-2 r}\right)\left(1-3^{1-2 r}\right)-2\right) .
$$

Now we can readily conclude Eq. (3).

Remark 7. Substituting $t=3 / 4$ and $l=2 r-1$ in Eq. (7), and using Eq. (4) we can obtain

$$
B_{2 r}=-\frac{2 r}{4\left(1+2^{-2 r}\left(1-2^{-(2 r-1)}\right)\right)} \sum_{k=1}^{2 r-1}(-1)^{k} \cdot \frac{S(2 r-1, k)}{k+1} \cdot\left(\frac{3}{4}\right)^{(k)} .
$$

The above is just a special case of Eq. (1) which was obtained in [6].

\section{References}

[1] H. W. Gould, Explicit formulas for Bernoulli numbers, Amer. Math. Monthly 79 (1972), $44-51$.

[2] A. Granville and Z. W. Sun, Values of Bernoulli polynomials, Pacific J. Math. 172 (1996), 117-137.

[3] G. H. Hardy, Ramanujan. Twelve Lectures on Subjects Suggested by His Life and Work, 3rd edition, Chelsea, 1978.

[4] S. K. Jha, Two new explicit formulas for the Bernoulli numbers, preprint, 2019. Available at https://arxiv.org/abs/1905.11216.

[5] S. K. Jha, A new explicit formula for the Bernoulli numbers in terms of the Stirling numbers of the second kind, preprint, 2019. Available at https://osf.io/95gkw.

[6] S. K. Jha, A new explicit formula for Bernoulli numbers involving the Euler number, Mosc. J. Comb. Number Theory 8 (2019), 385-387. 
[7] S. E. Landsburg, Stirling numbers and polylogarithms, preprint, 2009. Available at http://www. landsburg. com/query.pdf.

[8] L. Lewin, Polylogarithms and Associated Functions, North-Holland, 1981.

[9] J. Quaintance and H. W. Gould, Combinatorial Identities For Stirling Numbers: The Unpublished Notes Of H. W. Gould, World Scientific, 2015.

[10] Z. H. Sun, Congruences involving Bernoulli and Euler numbers, J. Number Theory 128 (2008), 280-312.

2010 Mathematics Subject Classification: Primary $11 \mathrm{~B} 68$.

Keywords: Bernoulli number, Stirling number of the second kind, polylogarithm function, Ramanujan's master theorem.

(Concerned with sequences A000367, A002445, A008277, A027641, and A $\underline{\text { A027642.) }}$

Received November 6 2019; revised versions received November 7 2019; January 19 2020; January 20 2020; January 23 2020; January 27 2020; February 10 2020; February 202020. Published in Journal of Integer Sequences, February 202020.

Return to Journal of Integer Sequences home page. 